\title{
Error probability approximation and codes selection in presence of multi-user interference for IR-UWB
}

\author{
Fatma Kharrat-Kammoun*, Philippe Ciblat*, and Christophe Le Martret ${ }^{\dagger}$ \\ *Institut TELECOM, TELECOM ParisTech, Paris, France \\ Email: fkharrat@enst.fr, philippe.ciblat@enst.fr \\ †Thalès Communications, Colombes, France \\ Email: christophe.le_martret@fr.thalesgroup.com
}

\begin{abstract}
An approximation for the Average Error Probability (AEP) of the Pulse Amplitude Modulation (PAM) Impulse Radio Ultra Wide Band (IR-UWB) systems in the presence of Multi-User Interference (MUI) is derived assuming that the spreading codes are fixed in time. The comparison of the proposed theoretical expression and the empirical result shows the accuracy of our approximation for both Direct-Sequence (DS) and Time-Hopping (TH) multiple access techniques. From this approximation, we deduce criterion that enable us to select the set of codes optimizing the performance in terms of the AEP.
\end{abstract}

\section{INTRODUCTION}

The exact Average Error Probability (AEP) has been expressed in [8] for TH IR-UWB system. Their derivations are based on characteristic function. The obtained AEP is obviously accurate but the obtained expression is still too much complex and does not provide any highlight about the influence of design parameters (such as the multi-user codes). Actually if we would like to obtain a simple AEP closed-from expression, we need to carefully approximate the multi-user interference (MUI) distribution. In [1], it was shown that the Generalized Gaussian Distribution (GGD) is a relevant choice to describe the MUI distribution. Assuming that the MUI is Generalized-Gaussian distributed, we derive an accurate closed form expression for the AEP in function of the multiple access codes for both TH and DS multiple access techniques. From the obtained AEP approximation, we are able to exhibit the criterion that the multiple access codes have to minimize in order to optimize the AEP for both access techniques. Minimizing such a criterion leads the optimal multiple access to satisfy some constraints that are characterized in this paper. The obtained results can be easily extended to the Pulse Position Modulation (PPM) TH IR-UWB system.

This paper is organized as follows. In Section II, we introduce the transmitted signal model for both TH and DS PAM IR-UWB systems, the channel model and the rake receiver structure. In Section III, we assume the codes to be fixed, and we derive the AEP in closed-form for the two analyzed systems using the Generalized Gaussian (GG) approximation whose parameters are evaluated as well in terms of the multiple access codes. In Section IV, we present the criterion that the codes have to satisfy in order to improve the performance. Section $\mathrm{V}$ is devoted to numerical illustrations. By empirical simulations, we validate our GGD based approximation and we inspect also the impact of the codes choice on the performance. We show the AEP significantly decreases when codes are selected as suggested in Section IV. Conclusions are given in Section VI.

\section{SIGNAL MODEL}

We consider a PAM IR-UWB system with either TH or DS as a code division multiple access technique. Let $N_{u}$ be the number of active users in the network. Each user transmits information asynchronously through a multipath channel. The transmitted signal from user $n$ can be expressed similarly for both DS and TH access techniques as follows:

$$
s_{n}(t)=\sum_{i=-\infty}^{\infty} d_{n}(i) \sum_{j=0}^{N_{c}-1} c_{n}(j) w\left(t-i T_{s}-j T_{c}-\theta_{n}\right)
$$

where

- $N_{c}$ is the number of chips per symbol,

- $T_{c}$ is the duration of one chip,

- $T_{s}$ is the symbol time,

- $w(t)$ is the normalized impulse of duration $T_{w} \ll T_{c}$,

- $d_{n}(i) \in\{-1,1\}$ are the information symbols of user $n$, assumed to be independent and identically distributed,

- $\left\{c_{n}(j)\right\}_{j=0}^{N_{c}-1}$ is the multiple access code, with $c_{n}(j) \in$ $\{-1,1\}$ for DS scheme and $c_{n}(j) \in\{0,1\}$ is the Developed Time Hopping (DTH) code [3], associated with user $n$,

- $\theta_{n}$ denotes the time asynchronism, assumed to be uniformly distributed random variable within $\left[0, T_{s}\right]$,

The receiver input signal is the sum of the attenuated and delayed transmitted signals from the different users. Its expression is given by

$$
r(t)=\sum_{n=1}^{N_{u}} \sqrt{P_{n}}\left(\sum_{k=1}^{N_{p}} A_{n}^{k} s_{n}\left(t-\tau_{n}^{k}\right)\right)+n(t)
$$

where $A_{n}^{k}$ and $\tau_{n}^{k}$ are the amplitude and the delay of the $k^{t h}$ path between the user $n$ and the receiver, $N_{p}$ is the number of paths, assumed to be the same for all the users, $P_{n}$ is the received power, and $n(t)$ is an additive zero-mean white Gaussian noise. 
The multipath channel model we employ is that proposed generally for UWB systems. The amplitude $A_{n}^{k}$ is usually assumed to be dependent on the delay $\tau_{n}^{k}$ as $A_{n}^{k}=a_{n}^{k} f\left(\tau_{n}^{k}\right)$, where $a_{n}^{k}$ are independent zero-mean random variables (rv) which account for the amplitude statistics and $f(\cdot)$ is a function which indicates the variation of the amplitude according to the delay. For sake of simplicity, we also consider that the channel impulse response is normalized, i.e., $\sum_{n=0}^{N_{p}}\left(A_{n}^{k}\right)^{2}=$ 1. The information about random received powers is provided by the set $\left\{P_{n}\right\}_{n=1, \cdots, N_{u}}$. The rv $\tau_{n}^{k}$ are assumed to be independent between users but are usually correlated for a given user. The distribution of the variables $\tau_{n}^{k}$ and $a_{n}^{k}$ are provided in the IEEE 802.15.3a standard [9]. When only one cluster is considered (which is not restrictive as mentioned in [10]), the delay $\tau_{n}^{k}$ follows a Poisson distribution. The attenuation $a_{n}^{k}=p_{n}^{k} \cdot \beta_{n}^{k}$, where $p_{n}^{k} \in\{ \pm 1\}$ are equiprobable and $\beta_{n}^{k}$ are log-normal rv. The function $f(\cdot)$ is defined by $f\left(\tau_{n}^{k}\right)=e^{-\tau_{n}^{k} / \gamma}$, where $\gamma$ is the path power-decay time.

Without loss of generality, the user of interest is assumed to be the user 1 . We consider the rake receiver of user 1 , commonly used for multipath channel systems, with $L_{r} \leq N_{p}$ fingers. We also assume that the receiver is synchronized, i.e., $\theta_{1}=0$. Thus, the rake receiver output is given by

$$
z=\sum_{\ell \in \mathcal{L}} A_{1}^{\ell} \int_{0}^{T_{s}} r\left(t+\tau_{1}^{\ell}\right) \cdot v_{1}(t) \mathrm{d} t
$$

where $v_{1}(t)=\sum_{j=0}^{N_{c}-1} c_{1}(j) w\left(t-j T_{c}\right)$ is the template signal associated with user 1 and $\mathcal{L}$ is the selected subset paths with $\operatorname{Card}(\mathcal{L})=L_{r}$. Using Eqs. (2)-(3), the rake receiver output is equal to

$$
z=\sum_{\ell \in \mathcal{L}} A_{1}^{\ell} \sum_{n=1}^{N_{u}} \sqrt{P_{n}} \sum_{k=1}^{N_{p}} A_{n}^{k} y_{k, \ell, n}\left(\theta_{n}\right)+\eta
$$

where $\eta=\sum_{\ell \in \mathcal{L}} A_{1}^{\ell} \int_{0}^{T_{s}} n\left(t+\tau_{1}^{\ell}\right) v_{1}(t) \mathrm{d} t$ is the filtered Gaussian noise, and

$$
\begin{aligned}
y_{k, \ell, n}\left(\theta_{n}\right) & =d_{n}\left(-Q_{n}^{k, \ell}\right)\left[\mathcal{C}_{1, n}^{+}\left(q_{n}^{k, \ell}\right) r\left(\epsilon_{n}^{k, \ell}\right)\right. \\
& \left.+\mathcal{C}_{1, n}^{+}\left(q_{n}^{k, \ell}+1\right) r\left(\epsilon_{n}^{k, \ell}-T_{c}\right)\right] \\
& +d_{n}\left(-Q_{n}^{k, \ell}-1\right)\left[\mathcal{C}_{1, n}^{-}\left(q_{n}^{k, \ell}\right) r\left(\epsilon_{n}^{k, \ell}\right)\right. \\
& \left.+\mathcal{C}_{1, n}^{-}\left(q_{n}^{k, \ell}+1\right) r\left(\epsilon_{n}^{k, \ell}-T_{c}\right)\right]
\end{aligned}
$$

with $Q_{n}^{k, \ell}=\left\lfloor\left(\theta_{n}+\tau_{n}^{k}-\tau_{1}^{\ell}\right) / T_{s}\right\rfloor, q_{n}^{k, \ell}=\left\lfloor\left(\theta_{n}+\tau_{n}^{k}-\tau_{1}^{\ell}-\right.\right.$ $\left.\left.Q_{n}^{k, \ell} T_{s}\right) / T_{c}\right\rfloor$, and $\epsilon_{n}^{k, \ell}=\theta_{n}+\tau_{n}^{k}-\tau_{1}^{\ell}-Q_{n}^{k, \ell} T_{s}-q_{n}^{k, \ell}$ which lies in $\left[0, T_{c}\right]$. We also put

$$
\begin{aligned}
\mathcal{C}_{m, n}^{-}(q) & =\sum_{k=0}^{q-1} c_{m}(k) c_{n}(k-q) \\
\mathcal{C}_{m, n}^{+}(q) & =\sum_{k=q}^{N_{c}-1} c_{m}(k) c_{n}(k-q)
\end{aligned}
$$

and $r(s)=\int_{-\infty}^{+\infty} w(t) w(t-s) \mathrm{d} t$. Notice that the rake receiver output given by Eq. (4) can be decomposed as [3]:

$$
z=z_{U}+z_{I}+z_{M}+\eta
$$

where,

- $z_{U}$ is the $U$ seful part of user 1 signal, and is given by $z_{U}=\sqrt{P_{1}} \sum_{\ell \in \mathcal{L}}\left(A_{1}^{\ell}\right)^{2} y_{\ell, \ell, 1}(0)$

- $z_{I}$ is the Inter-symbol interference from user 1 , and is given by $z_{I}=\sqrt{P_{1}} \sum_{\ell \in \mathcal{L}} A_{1}^{\ell} \sum_{k \neq \ell=1}^{N_{p}} A_{1}^{k} y_{k, \ell, 1}(0)$

- $z_{M}$ is the Multi-user interference, and is given by $z_{M}=$ $\sum_{\ell \in \mathcal{L}} A_{1}^{\ell} \sum_{n=2}^{N_{u}} \sqrt{P_{n}} \sum_{k=1}^{N_{p}} A_{n}^{k} y_{k, \ell, n}\left(\theta_{n}\right)$

Unlike $z_{I}$ and $z_{M}$, the useful part $z_{U}$ and the filtered noise $\eta$ do not depend on the multiple access codes. Fortunately, if the channel is short enough compared to symbol period, the term $z_{I}$ can be neglected by inserting a guard time [7]. However, the MUI can only be mitigated by a judicious choice of the multiple access codes. In the sequel, for sake of simplicity, on the one hand, we assume that $z_{I}=0$, and on the other hand, we consider the set of received powers fixed, i.e., the obtained closed-form expression will depend on the realization of $\left\{P_{n}\right\}_{n=1, \cdots, N_{u}}$.

\section{AEP APPROXIMATION BASED ON GENERALIZED GAUSSIAN DISTRIBUTION}

The first works dealing with the performance of UWB systems assumed that the MUI was a Gaussian distributed random variable. Later, it has been proved in [5], [6] that the Gaussian approximation is not valid in many cases. Recently, it has been proposed to use the GGD to describe the MUI distribution in TH IR-UWB system [1] in AWGN context. By simulation, we remarked that the MUI can still be well modeled by GGD when IEEE 802.15.3a standard based multipath channels (described in Section II) are implemented. We remind that the GGD writes as follows [4]:

$$
p(x)=\frac{\sqrt{\Gamma_{c}(3 / \alpha)}}{2 \sigma \sqrt{\Gamma_{c}(1 / \alpha)} \Gamma_{c}(1+1 / \alpha)} e^{-\left|\frac{\sqrt{\Gamma_{c}(3 / \alpha)}}{\sigma \sqrt{\Gamma_{c}(1 / \alpha)}} x\right|^{\alpha}}
$$

where $\sigma^{2}=\mathbb{E}\left[x^{2}\right]$ is the variance, $\alpha>0$ is the so-called shape parameter, and $x \mapsto \Gamma_{c}(x)$ is the Gamma function. Remark that when $\alpha=2, p(x)$ corresponds to a Gaussian distribution. In the sequel, given the set of received powers, we derive the AEP when the MUI is assumed to be GG distributed.

\section{A. AEP approximation based on $G G D$}

First of all, notice that the useful signal $z_{U}$ in Eq. (8) is equal to $z_{U}=d_{1}(0) \sqrt{P_{1}} N_{s}$ where $N_{s}$ is the repetition factor ( $N_{s}=N_{c}$ for DS-UWB system; $N_{s}=N_{f}$ for TH-UWB system where $N_{f}$ is the number of frame). Then, since the GGD is symmetric and the PAM modulation is equilikely, the AEP is given by:

$$
\bar{P}_{e}=\operatorname{Prob}\left(\nu>\sqrt{P_{1}} N_{s}\right)=\int_{\sqrt{P_{1}} N_{s}}^{+\infty} p_{\nu}(x) d x
$$

where $\nu=z_{M}+\eta$ is the term disturbing the decision and where $p_{\nu}(x)$ is its distribution.

As $z_{M}$ is assumed to be GG distributed and as $\eta$ is Gaussian distributed, i.e., GG distributed, we know that $\nu$ is also well approximated by a GGD. Indeed, in [2], one has been mentioned that the sum of two GG distributed variables can be approximated by a GG distributed variable as well. 
Consequently, the distribution of $\nu$ is described by Eq. (9) whose the shape parameter and variance are $\alpha$ and $\sigma^{2}$ respectively. Note that the expressions of $\alpha$ and $\sigma^{2}$ in terms of multiple access codes will be calculated in Section III-B. By replacing $p_{\nu}(x)$ in Eq. (10) with its expression in Eq. (9) and by doing tedious but straightforward algebraic manipulations, we get

$$
\bar{P}_{e}=\frac{1}{2 \alpha \Gamma_{c}(1+1 / \alpha)} \Gamma_{i}\left[\frac{1}{\alpha},\left(\frac{N_{s} \sqrt{\Gamma_{c}(3 / \alpha)}}{\sigma \sqrt{\Gamma_{c}(1 / \alpha)}}\right)^{\alpha}\right]
$$

where $\Gamma_{i}[.,$.$] is the so-called incomplete Gamma function$ defined by $\Gamma_{i}[a, x]=\int_{x}^{+\infty} t^{a-1} \exp (-t) \mathrm{d} t$.

\section{B. GGD parameters vs the multiple access codes}

In the sequel, we denote by $\alpha_{M}$ and $\sigma_{M}^{2}$ the shape parameter and variance of signal $z_{M}$ respectively. Terms $\alpha_{\eta}=2$ and $\sigma_{\eta}^{2}$ stand for the shape parameter and variance of noise $\eta$ respectively. Let us now focus on the derivation of $\sigma^{2}$ and $\alpha$. As $z_{M}$ and $\eta$ are zero mean, we have

$$
\sigma^{2}=\sigma_{M}^{2}+\sigma_{\eta}^{2}
$$

As mentioned [1], the shape parameter $\alpha$ of a GGD is related to the $4^{\text {th }}$ and $2^{\text {th }}$ order moment as follows

$$
\alpha=F^{(-1)}\left(\frac{D^{4}}{\sigma^{4}}\right)
$$

where $D^{4}=\mathbb{E}\left[\nu^{4}\right]$, and where $F^{(-1)}($.$) is the reciprocal$ function of $x \mapsto F(x)=\Gamma_{c}(5 / x) \Gamma_{c}(1 / x) / \Gamma_{c}^{2}(3 / x)$. Let $D_{M}^{4}=\mathbb{E}\left[z_{M}^{4}\right]$ and notice that $\mathbb{E}\left[\eta^{4}\right]=3 \sigma_{\eta}^{4}$. Like the $2^{\text {th }}$ order moment, the $4^{\text {th }}$ order moment of $\nu$ can be expressed in function of those of $z_{M}$ and $\eta$ as

$$
D^{4}=D_{M}^{4}+3 \sigma_{\eta}^{4}+6 \sigma_{M}^{2} \sigma_{\eta}^{2} .
$$

In order to determine perfectly the statistics of $\nu$ in terms of the multiple access codes, we only need to derive $\sigma_{M}^{2}$ and $D_{M}^{4}$ in terms of the multiple access codes. In [1], the average of $\sigma_{M}^{2}$ and $D_{M}^{4}$ over all the TH multiple access codes were evaluated. In our work, we remind that the multiple access codes are fixed since we would to select them according to the minimization of the obtained AEP approximation. Notice that the expectation for deriving the $2^{\text {th }}$ and $4^{\text {th }}$ order moments is achieved over the channel amplitude $a_{n}^{k}$, the symbol $d_{n}$, the asynchronism $\theta_{n}$ and the delay $\tau_{n}$ according to this order.

1) Closed-form expression of $\sigma_{M}^{2}:=\mathbb{E}_{a, d, \theta, \tau}\left[z_{M}^{2}\right]$ : in [3] dedicated to TH-UWB system, we have

$$
\sigma_{M}^{2}=\frac{\gamma_{1}}{T_{s}} \sum_{n=2}^{N_{u}} P_{n} \Psi_{n} \sum_{q=0}^{N_{c}-1}\left[\mathcal{C}_{1, n}^{-2}(q)+\mathcal{C}_{1, n}^{+2}(q)\right]
$$

where $\gamma_{1}=\int r^{2}(t) \mathrm{d} t, \Psi_{n}=\sum_{\ell \in \mathcal{L}} \mathbb{E}_{\tau}\left[I_{1}^{\ell}\right] \sum_{k=1}^{N_{p}} \mathbb{E}_{\tau}\left[I_{n}^{k}\right]$ with $I_{n}^{k}=\mathbb{E}_{a}\left[\left(A_{n}^{k}\right)^{2}\right]$.

Thanks to Eq. (1), one can see that the MUI can be similarly represented for TH-UWB and DS-UWB when employing PAM modulation. Consequently the variance given by Eq. (15) remains valid for DS-UWB system.
2) Closed-form expression of $D_{M}^{4}:=\mathbb{E}_{a, d, \theta, \tau}\left[z_{M}^{4}\right]$ : the MUI can be decomposed as $z_{M}=\sum_{n=2}^{N_{u}} z_{M, n}$ where $z_{M, n}=$ $\sqrt{P_{n}} \sum_{k=1}^{N_{p}} A_{n}^{k} y_{k, \ell, n}\left(\theta_{n}\right)$ is the interference associated with user $n$. Since the symbols $d_{n}$ and $d_{n}^{3}$ are zero-mean, the expectation of $z_{M}^{4}$ over the amplitude $a$ and the symbol $d$ is given by

$$
\mathbb{E}_{a, d}\left[z_{M}^{4}\right]=\sum_{n=2}^{N_{u}} \mathbb{E}_{a, d}\left[z_{M, n}^{4}\right]+6 \sum_{\substack{n, m=2 \\ n \neq m}}^{N_{u}} \mathbb{E}_{a, d}\left[z_{M, n}^{2}\right] \mathbb{E}_{a, d}\left[z_{M, m}^{2}\right]
$$

As the time-support of $r(\cdot)$ is much less than $T_{c}$, we have $r^{p}(\epsilon) r^{q}\left(\epsilon-T_{c}\right)=0, \forall p, q$. Consequently, we get

$$
\begin{aligned}
\mathbb{E}_{a, d}\left[z_{M, n}^{4}\right] & =P_{n}^{2} \sum_{\substack{k=1 \\
\ell \in \mathcal{L}}}^{N_{p}} J_{1}^{\ell} J_{n}^{k}\left[\left(\mathcal{C}_{1, n}^{+4}\left(q_{n}^{k, \ell}\right)+\mathcal{C}_{1, n}^{-4}\left(q_{n}^{k, \ell}\right)\right) r^{4}\left(\epsilon_{n}^{k, \ell}\right)\right. \\
& +\left(\mathcal{C}_{1, n}^{+4}\left(q_{n}^{k, \ell}+1\right)+\mathcal{C}_{1, n}^{-4}\left(q_{n}^{k, \ell}+1\right)\right) r^{4}\left(\epsilon_{n}^{k, \ell}-T_{c}\right) \\
& +6 \mathcal{C}_{1, n}^{+2}\left(q_{n}^{k, \ell}\right) \mathcal{C}_{1, n}^{-2}\left(q_{n}^{k, \ell}\right) r^{4}\left(\epsilon_{n}^{k, \ell}\right) \\
& \left.+6 \mathcal{C}_{1, n}^{+2}\left(q_{n}^{k, \ell}+1\right) \mathcal{C}_{1, n}^{-2}\left(q_{n}^{k, \ell}+1\right) r^{4}\left(\epsilon_{n}^{k, \ell}-T_{c}\right)\right]
\end{aligned}
$$

with $J_{n}^{k}=\mathbb{E}_{a}\left[\left(A_{n}^{k}\right)^{4}\right]$.

The expectation of $\mathbb{E}_{a, d}\left[z_{M, n}^{4}\right]$ over the uniform variable $\theta_{n}$ is obtained by $\mathbb{E}_{a, d, \theta}\left[z_{M, n}^{4}\right]=\frac{1}{T_{s}} \int_{T_{s}} \mathbb{E}_{a, d}\left[z_{M, n}^{4}\right] d \theta$. By writing the integral over $\left[0, T_{s}\right]$ as a sum of integrals over the subinterval $\left[0, T_{c}\right]$, and by taking into account the periodicity of the multiple access codes, we find

$$
\begin{aligned}
\mathbb{E}_{a, d, \theta}\left[z_{M, n}^{4}\right] & =\frac{\gamma_{2}}{T_{s}} P_{n}^{2} \sum_{\substack{k=1 \\
\ell \in \mathcal{L}}}^{N_{p}} J_{1}^{\ell} J_{n}^{k} \\
& \times \sum_{q=0}^{N_{c}-1}\left[\mathcal{C}_{1, n}^{+4}(q)+\mathcal{C}_{1, n}^{-4}(q)+6 \mathcal{C}_{1, n}^{+2}(q) \mathcal{C}_{1, n}^{-2}(q)\right]
\end{aligned}
$$

with $\gamma_{2}=\int r_{w w}^{4}(t) \mathrm{d} t$.

Finally, using previous equalities, averaging $\mathbb{E}_{a, d, \theta}\left[z_{M, n}^{4}\right]$ over the delays $\tau_{n}^{k}-\tau_{1}^{\ell}$, and reminding that $\sigma_{M}^{2}=$ $\sum_{n=2}^{N_{u}} \mathbb{E}_{a, d, \theta, \tau}\left[z_{M, n}^{2}\right]$ leads to the following expression for the $4^{\text {th }}$ order moment

$$
\begin{aligned}
D_{M}^{4} & =\frac{\gamma_{2}}{T_{s}} \sum_{n=2}^{N_{u}} P_{n}^{2} \Phi_{n}\left[\frac{3\left(N_{u}-2\right)}{N_{u}-1} \sigma_{M}^{4}\right. \\
& \left.+\sum_{q=0}^{N_{c}-1} \mathcal{C}_{1, n}^{+4}(q)+\mathcal{C}_{1, n}^{-4}(q)+6 \mathcal{C}_{1, n}^{+2}(q) \mathcal{C}_{1, n}^{-2}(q)\right]
\end{aligned}
$$

where $\Phi_{n}=\sum_{\ell \in \mathcal{L}} \mathbb{E}_{\tau}\left[J_{1}^{\ell}\right] \sum_{k=1}^{N_{p}} \mathbb{E}_{\tau}\left[J_{n}^{k}\right]$ and where $\sigma_{M}^{2}$ is given by Eq. (15).

\section{Multiple ACCESS CODES Minimizing THE AEP}

Given $N_{s}$ and the receiver powers, the AEP (see Eq. (11)) depends only on $\alpha$ and $\sigma^{2}$. For a given $\sigma^{2}$, one can remark that $\bar{P}_{e}$ decreases when $\alpha$ increases at high SINR. Therefore, in order to minimize the performance, i.e., the AEP, we have to select the codes that minimize $\sigma^{2}$ and then maximize $\alpha$. Thanks to Eq. (13) and the monotonic decreasing property of $F^{(-1)}($.$) , maximizing \alpha$ is equivalent to minimizing $D^{4}$ 
when $\sigma^{2}$ is fixed. Notice that the high SINR assumption is not restrictive since we would like to improve the error floor occuring in the Rake receiver. Thanks to Eqs. (12)(14) and thanks to the independence of $\sigma_{\eta}^{2}$ with respect to the multiple access codes codes, minimizing $\sigma^{2}$ and $D^{4}$ with respect to the multiple access boils down to minimizing $\sigma_{M}^{2}$ and $D_{M}^{4}$ with respect to the multiple access codes. Unlike the previous sections of this paper, we need hereafter distinguish the multiple access codes selection for TH-UWB and DSUWB systems since the codes belong to $\{0,1\}$ and $\{-1,1\}$ in $\mathrm{TH}$ and DS case respectively.

\section{A. Optimal Developed Time Hopping codes}

Before going further, we introduce the following proposition for which we omit the proof due to the lack of space. Proof can be done similarly to the proof of Theorem 1 in [3].

Proposition 1: Let us consider a pair of DTH code $\left(c_{m}, c_{n}\right)$ that satisfies $\sum_{q=0}^{N_{c}-1} \mathcal{C}_{m, n}^{+2}(q)+\mathcal{C}_{m, n}^{-2}(q)=N_{s}^{2}$, then

$$
\sup _{q} \mathcal{C}_{m, n}^{+}(q)=1 \text { and } \sup _{q} \mathcal{C}_{m, n}^{-}(q)=1
$$

We are then able to state Theorem 1 which characterize the Developed Time Hopping codes minimizing the AEP.

Theorem 1: The AEP of user of interest 1 is minimum, if and only if, the set of pair of DTH codes $\left\{\left(c_{1}, c_{n}\right), n=\right.$ $\left.2, \ldots, N_{u}\right\}$, satisfies

$$
\sum_{q=0}^{N_{c}-1}\left(\mathcal{C}_{1, n}^{+}(q)+\mathcal{C}_{1, n}^{-}(q)\right)^{2}=N_{s}^{2}
$$

Notice that, in [3], the authors suggest to select the DTH codes minimizing the variance, i.e., satisfying $\sum_{q=0}^{N_{c}-1}\left(\mathcal{C}_{1, n}^{+2}(q)+\right.$ $\left.\mathcal{C}_{1, n}^{-2}(q)\right)=N_{s}^{2}$ which corresponds to a larger set of codes than the set of codes verifying Eq. (18).

Proof: We aim to identify the pair of codes that minimizes both $\sigma_{M}^{2}$ and $D_{M}^{4}$ given by Eqs. (15) and (16) respectively. In [3], it has been already proven that a pair of DTH codes minimizes $\sigma_{M}^{2}$ if and only if $\sum_{q=0}^{N_{c}-1} \mathcal{C}_{1, n}^{+2}(q)+$ $\mathcal{C}_{1, n}^{-2}(q)=N_{s}^{2}$. Let us consider a pair of codes that satisfies this last condition and let us prove that $D_{M}^{4}$ is minimal if and only if $\sum_{q=0}^{N_{c}-1} \mathcal{C}_{1, n}^{+}(q) \mathcal{C}_{1, n}^{-}(q)=0$. Using Eq. (17) and noting that $\mathcal{C}_{1, n}^{-}(q), \mathcal{C}_{1, n}^{+}(q) \geq 0$, we can deduce that $\sum_{q=0}^{N_{c}-1} \mathcal{C}_{1, n}^{+4}(q)+$ $\mathcal{C}_{1, n}^{-4}(q)$ is minimal as well and is equal to $N_{s}^{2}$. Hence, $D_{M}^{4}$ is minimal if and only if $\sum_{q=0}^{N_{c}-1} \mathcal{C}_{1, n}^{+2}(q) \mathcal{C}_{1, n}^{-2}(q)=0$, or equivalently, $\sum_{q=0}^{N_{c}-1} \mathcal{C}_{1, n}^{+}(q) \mathcal{C}_{1, n}^{-}(q)=0$ due to Eq. (17) for the DTH codes minimizing the variance and due to the positivity of $\mathcal{C}_{1, n}^{-}(q)$ and $\mathcal{C}_{1, n}^{+}(q)$. This concludes the proof.

\section{B. Optimal Direct Sequence codes}

Before exhibiting the Optimal Direct Sequence codes in Theorem 2, we introduce two preliminary propositions.

Proposition 2: Let $\left(c_{m}, c_{n}\right)$ be two Direct Sequence codes of length $N_{c}$. We have

$$
\sum_{q=0}^{N_{c}-1} \mathcal{C}_{m, n}^{+2}(q)+\mathcal{C}_{m, n}^{-2}(q) \geq N_{c}
$$

Proof: Given Eqs. (6)-(7), we show that $\mathcal{C}_{m, n}^{-}(q)$ and $\mathcal{C}_{m, n}^{+}(q)$ consist of $q$ and $N_{c}-q$ terms respectively. Each term belongs to $\{ \pm 1\}$. When $N_{c}$ is odd, $q$ and $N_{c}-q$ does not have the same parity. Consequently, when $q$ is even, $\mathcal{C}_{m, n}^{-}(q)$ is lower-bounded by 0 and $\mathcal{C}_{m, n}^{+}(q)$ is lower-bounded by 1 which implies that $\mathcal{C}_{m, n}^{+2}(q)+\mathcal{C}_{m, n}^{-2}(q)$ is lower-bounded by 1 . When $q$ is odd, we just have to permute the role of $\mathcal{C}_{m, n}^{-}(q)$ and $\mathcal{C}_{m, n}^{+}(q)$. Then we deduce immediately that $\sum_{q=0}^{N_{c}-1} \mathcal{C}_{m, n}^{+2}(q)+$ $\mathcal{C}_{m, n}^{-2}(q)$ is lower bounded by $N_{c}$ the number of terms in the sum. When $N_{c}$ is even, similar proof can be done.

Proposition 3: Let $\left(c_{1}, c_{n}\right)$ be a pair of Direct Sequence code satisfying $\sum_{q=0}^{N_{c}-1} \mathcal{C}_{1, n}^{+2}(q)+\mathcal{C}_{1, n}^{-2}(q)=N_{c}$.

- In the case of even $N_{c}:\left|\mathcal{C}_{1, n}^{+}(q)\right|=\left|\mathcal{C}_{1, n}^{-}(q)\right|=0$ if $q$ is even; and $\left|\mathcal{C}_{1, n}^{+}(q)\right|=\left|\mathcal{C}_{1, n}^{-}(q)\right|=1$ if $q$ is odd.

- In the case of odd $N_{c}:\left|\mathcal{C}_{1, n}^{+}(q)\right|=1$ and $\left|\mathcal{C}_{1, n}^{-}(q)\right|=0$ if $q$ is odd; and $\left|\mathcal{C}_{1, n}^{+}(q)\right|=0$ and $\left|\mathcal{C}_{1, n}^{-}(q)\right|=1$ if $q$ is even.

Due to the lack of space, the proof of Proposition 3 is omitted. Nevertheless the proof can be done in similar way of those of Proposition 2.

Theorem 2: The AEP of user of interest 1 is minimum, if and only if, the set of pair of DS codes $\left\{\left(c_{1}, c_{n}\right), n=\right.$ $\left.2, \ldots, N_{u}\right\}$, satisfies

$$
\sum_{q=0}^{N_{c}-1} \mathcal{C}_{1, n}^{+2}(q)+\mathcal{C}_{1, n}^{-2}(q)=N_{c}
$$

Unlike TH scheme, we also see that minimizing jointly the variance and the shape parameter of MUI distribution is equivalent to minimizing the variance only, in DS scheme context.

Proof: Due to lack of space, we only prove that $\sum_{q=0}^{N_{c}-1} \mathcal{C}_{1, n}^{+2}(q)+\mathcal{C}_{1, n}^{-2}(q)=N_{s}$ yields that $\sigma_{M}^{2}$ and $D_{M}^{4}$ are minimal. The proof of the reverse implication can be easily done by using Propositions 2 and 3 . Let $\left\{\left(c_{1}, c_{n}\right)\right\}$ be a set of pair of DS code satisfying Eq. (20). Thanks to Eq. (19), we have that the variance $\sigma_{M}^{2}$ (given by Eq. (15)) is minimal. Let us now focus on $D_{M}^{4}$ given by Eq. (16). By means of Proposition 3, one can easily check that $\sum_{q=0}^{N_{c}-1} \mathcal{C}_{1, n}^{+4}(q)+\mathcal{C}_{1, n}^{-4}(q)$ is minimal and is equal to $N_{c}$. The second term of $D_{M}^{4}$, given by $\sum_{q=0}^{N_{c}-1} \mathcal{C}_{1, n}^{+2}(q) \mathcal{C}_{1, n}^{-2}(q)$, is equal to $N_{c} / 2$ if $N_{c}$ is even and 0 otherwise, and thus identical for any code minimizing the variance. Therefore, Eq. (20) leads to the joint minimization of $\sigma_{M}^{2}$ a,d $D_{M}^{4}$.

\section{NUMERICAL RESULTS}

We consider an AWGN channel and a normalized Gaussian impulse

$$
w(t)=A_{w} \sqrt{\frac{2}{\pi}} \frac{\cos \left(2 \pi f_{c} t\right)}{\lambda} e^{-\frac{t^{2}}{2 \lambda^{2}}}
$$

with $A_{w}$ is a normalized factor such that $\int_{-\infty}^{+\infty} w^{2}(t) \mathrm{d} t=1$, $f_{c}=6.85 \mathrm{GHz}$ and $\lambda=9.107 \times 10^{-2} \mathrm{~ns}$.

In Figures (1) and (2) we compared the theoretical AEP approximation given by Eq. (11) (displayed in dotted lines) to the empirical Bit Error Rate (displayed in solid lines) with 


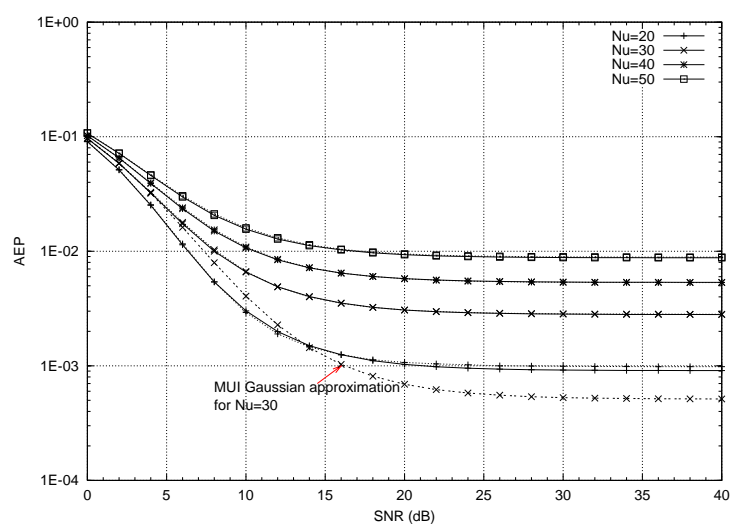

Fig. 1. Theoretical AEP and BER for PAM TH-UWB system with $N_{c}=16$, $N_{s}=4, T_{c}=3 \mathrm{~ns}$ and random codes.

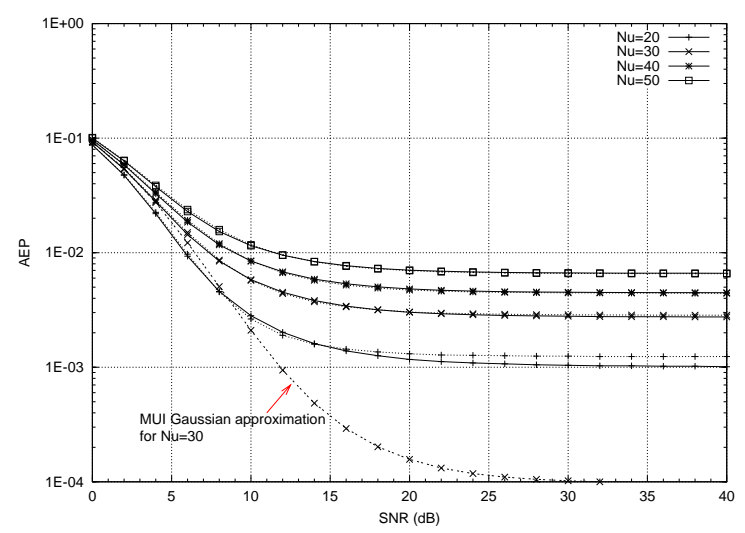

Fig. 2. Theoretical AEP and BER for PAM DS-UWB system with $N_{c}=$ $N_{s}=6, T_{c}=8 \mathrm{~ns}$ and random codes.

different values of $N_{u}$ for TH-UWB and DS-UWB systems respectively. The symbol time is equal to $T_{s}=48 \mathrm{~ns}$ for both systems. For TH system, the number of chips $N_{c}$ is equal to 16 and the repetition factor $N_{s}$ is equal to 4 . For DS system, we have $N_{s}=N_{c}=6$. Both figures show the accuracy of our approximation when the codes are chosen at random for the different $N_{u}$ values. The error probability with the Gaussian approximation ( $\alpha=2$ in Eq. (11)) is also plotted in both figures for $N_{u}=30$. The Gaussian approximation clearly underestimates the error probability for TH-UWB and DSUWB systems as already observed in [5].

Let us now consider a PAM TH-UWB system with $N_{u}=30$ active users, the symbol time $T_{s}=72 \mathrm{~ns}$, the number of chips is $N_{c}=24$ and the repetition factor $N_{s}=4$. In Figure 3, we inspect the impact of the multiple access codes on the performance. We are interesting to three cases: case 1 corresponds to random codes, case 2 corresponds to the codes minimizing the MUI variance $\sigma_{M}^{2}$ as done in [3], and case 3 corresponds to the codes verifying Eq. (18). By comparing these different cases, we notice that the last case leads to the best performance. The selection of the codes that minimize only the variance does not guarantee a minimal error probability. These codes nevertheless improve the performance

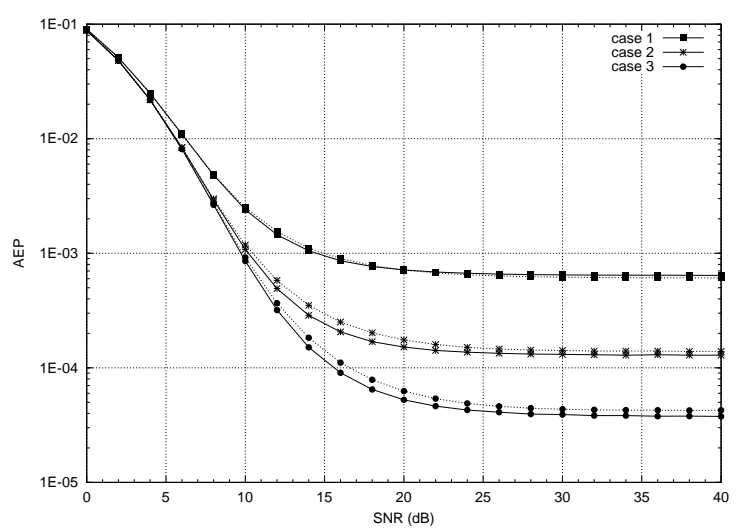

Fig. 3. Performance wrt the codes properties for PAM TH-UWB system with $N_{c}=24, N_{s}=4, T_{c}=3 \mathrm{~ns}$ and $N_{u}=30$.

with respect to the random codes.

\section{CONCLUSIONS}

An accurate error probability approximation for both PAM TH and DS IR-UWB systems has been derived assuming the MUI distribution is well modeled by GGD for any set of fixed multiple access codes. We then were able to select the multiple access codes minimizing the error probability. Numerical results show significant gains while selecting the appropriate codes in TH context.

\section{REFERENCES}

[1] J. Fiorina and D. Domenicali, "Revisiting TH-IR-UWB performance limits dependency on essential system parameters using the Generalized Gaussian Approximation," in Proc. IEEE International Conference on Ultra-Wideband, pp. 751-754, Sept. 2007.

[2] W. Niehsen, "Robust Kalman filtering with generalized Gaussian measurement noise," IEEE Trans. on Aerospace and Electronic Systems, vol. 38, no. 4, pp. 1409-1412, Oct. 2002.

[3] C.J. Le Martret, A.-L. Deleuze, and P. Ciblat, "Optimal time-hopping codes for multi-user interference mitigation in ultra-wide bandwidth impulse radio," IEEE Trans. on Wireless Communications, vol. 5, no. 6, pp. 1516-1525, June 2006.

[4] S. M. Kay, Fundamentals of Statistical Signal Processing: Detection Theory, Englewood Cliffs: Prentice Hall 1998.

[5] G. Durisi and G. Romano, "On the validity of Gaussian approximation to characterize the multiuser capacity of UWB TH-PPM," in Proc. IEEE Conference on Ultra Wideband Systems and Technologies., pp. 157-162, May 2002.

[6] J. Fiorina and W. Hachem, "On the asymptotic distribution of the correlation receiver output for time-hopped UWB signals," IEEE Trans. on Signal Processing, vol. 54, no. 7, pp. 2529-2545, July 2006.

[7] M. Z. Win and R. A. Scholtz, "Ultra-wide bandwidth time-hopping spread-spectrum impulse radio for wireless multiple-access communications," IEEE Trans. on Communications, vol. 48, no. 4, pp. 679-691, April 2000 .

[8] B. Hu and N.C. Beaulieu, "Accurate performance evaluation of timehopping and direct-sequence UWB systems in multi-user interference," IEEE Trans. on Communications, vol. 53, no. 6, pp. 1053-1062, June 2005.

[9] A. F. Molish, J. R. Foerster, and M. Pendergrass, "Channel models for ultrawideband personal area networks," IEEE Wireless Communications, vol. 10, Dec. 2003.

[10] R. D. Wilson, R. A. Scholtz, "Comparison of CDMA and modulation schemes for UWB radio in a multipath environment," in Proc. of IEEE Global Telecomm. Conf. (GLOBECOM), vol. 2, pp. 754-758, Dec. 2003. 